KU-TP 055

\title{
Unitarity versus Renormalizability of Higher Derivative Gravity in $3 D$
}

\author{
Kenji Muneyuki and Nobuyoshi Ohta ${ }^{1}$ \\ Department of Physics, Kinki University, Higashi-Osaka, Osaka 577-8502, Japan
}

\begin{abstract}
It has been suggested that new massive gravity with higher order terms in the curvature may be renormalizable and thus a candidate for renormalizable quantum gravity. We show that threedimensional gravity that contains quadratic scalar curvature and Ricci tensor is renormalizable, but those theories with special relation between their coefficients including new massive gravity are not.
\end{abstract}

\footnotetext{
${ }^{1}$ e-mail address: ohtan@phys.kindai.ac.jp
} 


\section{Introduction}

Constructing quantum theory of gravity is one of the most important problems in theoretical physics. It has been known for some time that gravity is renormalizable in four dimensions if one includes higher derivative terms [1]. However there is a price. The unitarity of the theory, which is one the most important properties of any physical theory, is not preserved. So the theory has not been taken very seriously.

Recently a very interesting proposal has been made that the addition of such higher order terms to three-dimensional gravity can keep the theory unitary and possibly renormalizable if the coefficients are chosen appropriately [2]. The usual Einstein gravity does not have any propagating mode in three dimensions, but the addition of these terms introduces propagating massive graviton around flat Minkowski and curved maximally symmetric spaces (anti-de Sitter and de Sitter spaces). A similar theory of massive graviton with higher derivative LorentzChern-Simons (LCS) term has long been known as topologically massive theory [3], but the theory violates parity. In contrast, the new theory is a parity preserving theory, and it is called new massive gravity. Since then, various aspects of the theory have been investigated. Linearized excitations in the field equations were studied in [4]. Unitarity is proven for Minkowski space in $[5,6,7]$, whereas it is discussed in [8] for maximally symmetric spaces. A complete classification of the unitary theory for the most general action with arbitrary coefficients of all possible terms is given in [9]. A partial result of the unitarity condition on the flat Minkowski space is known for the usual sign of the Einstein theory [10].

Though these kinds of theories have their own significance, it is also known that such higher order terms are present in the low-energy effective theories of superstrings. In this circumstance, these terms are regarded just as perturbative corrections to the lowest order terms, and are not considered to be modes to be quantized together. However, it is more appropriate to consider these terms together if one would like to understand the quantum theory of gravity. Also, there is some ambiguity in such theories due to the field redefinition. If the approach of requiring unitarity and renormalizability determines the coefficients to certain extent, it may cast some light on the superstrings themselves.

With such higher order terms, one may expect that the theory is renormalizable. In fact, it has been argued that the topological massive gravity may be renormalizable if suitable regularization is given [11], but this theory breaks parity and the standard gauge-invariant regularization such as dimensional regularization cannot be used. More recently, it was claimed that the new massive gravity is renormalizable [12]. However, there seem to be flaws in this argument, and we will discuss where it fails. There are also several arguments against renormalizability [13], and this is still under debate. It is important to settle the issue of whether the gravitational theory is unitary and renormalizable or not. In this paper we critically examine the renormalizability of the three-dimensional gravity with quadratic curvature terms and try to clarify the situation.

Following the general discussions of renormalizability of four-dimensional higher derivative gravity using Becchi-Rouet-Stora-Tyutin (BRST) symmetry [1], we study the quantum property of the three-dimensional theory. We examine the theory with arbitrary coefficients for scalar and Ricci curvature squared but without a LCS term because we assume the gauge-invariant dimensional regularization in our analysis, which may not be used for parity-violating theory. This analysis shows that the theory is renormalizable for general coefficients of these terms. However, we find that there are two important exceptions to this. The renormalizability fails precisely for the cases of unitary theories around flat Minkowski space, the new massive gravity 
and $R^{2}$ theory. Thus, unfortunately, the unitarity and renormalizability do not seem to be compatible. We would like to emphasize that it is important to settle this question in the context of searching for quantum gravity.

\section{Higher Derivative Gravity in 3D}

Let us start with the action

$$
\begin{aligned}
S & =\frac{1}{\kappa^{2}} \int d^{3} x \sqrt{-g}\left[\sigma R+\alpha R^{2}+\beta R_{\mu \nu}^{2}\right] \\
& \equiv \frac{1}{\kappa^{2}} \int d^{3} x \mathcal{L}_{G M G}
\end{aligned}
$$

where $\kappa^{2}$ is the three-dimensional gravitational constant, $\alpha, \beta$ and $\sigma(=0, \pm 1)$ are constants. Though we can have topological mass term given by the gravitational LCS term, we do not consider it in this paper for simplicity.

\subsection{Propagator}

We define the fluctuation around the Minkowski background by

$$
\tilde{g}^{\mu \nu} \equiv \sqrt{-g} g^{\mu \nu}=\eta^{\mu \nu}+\kappa h^{\mu \nu} .
$$

Substituting (2) into our action (1), we find the quadratic term is given by

$$
\begin{gathered}
\mathcal{L}_{2}=\frac{1}{4} h^{\mu \nu}\left[P^{(2)}(\beta \square+\sigma)+P^{(0, s)}\{(8 \alpha+3 \beta) \square-\sigma\}+2 P^{(0, w)}\{(8 \alpha+3 \beta) \square-\sigma\}\right. \\
\left.+\sqrt{2}\left(P^{(0, s w)}+P^{(0, w s)}\right)\{(8 \alpha+3 \beta) \square-\sigma\}\right]_{\mu \nu, \rho \sigma} \square h^{\rho \sigma},
\end{gathered}
$$

where we have defined the projection operators as [10]

$$
\begin{aligned}
P_{\mu \nu, \rho \sigma}^{(2)} & =\frac{1}{2}\left(\theta_{\mu \rho} \theta_{\nu \sigma}+\theta_{\mu \sigma} \theta_{\nu \rho}-\theta_{\mu \nu} \theta_{\rho \sigma}\right) \\
P_{\mu \nu, \rho \sigma}^{(1)} & =\frac{1}{2}\left(\theta_{\mu \rho} \omega_{\nu \sigma}+\theta_{\mu \sigma} \omega_{\nu \rho}+\theta_{\nu \rho} \omega_{\mu \sigma}+\theta_{\nu \sigma} \omega_{\mu \rho}\right) \\
P_{\mu \nu, \rho \sigma}^{(0, s)} & =\frac{1}{2} \theta_{\mu \nu} \theta_{\rho \sigma}, \quad P_{\mu \nu, \rho \sigma}^{(0, w)}=\omega_{\mu \nu} \omega_{\rho \sigma} \\
P_{\mu \nu, \rho \sigma}^{(0, s w)} & =\frac{1}{\sqrt{2}} \theta_{\mu \nu} \omega_{\rho \sigma}, \quad P_{\mu \nu, \rho \sigma}^{(0, w s)}=\frac{1}{\sqrt{2}} \omega_{\mu \nu} \theta_{\rho \sigma}
\end{aligned}
$$

with

$$
\theta_{\mu \nu}=\eta_{\mu \nu}-\frac{\partial_{\mu} \partial_{\nu}}{\square}, \quad \omega_{\mu \nu}=\frac{\partial_{\mu} \partial_{\nu}}{\square}
$$

$P^{(2)}, P^{(1)}, P^{(0, s)}$ and $P^{(0, w)}$ are the projection operators onto spin 2,1 and 0 parts, and they satisfy the completeness relation

$$
\left(P^{(2)}+P^{(1)}+P^{(0, s)}+P^{(0, w)}\right)_{\mu \nu, \rho \sigma}=\frac{1}{2}\left(\eta_{\mu \rho} \eta_{\nu \sigma}+\eta_{\mu \sigma} \eta_{\nu \sigma}\right),
$$

on the symmetric second-rank tensors. 
The BRST transformation for the fields is found to be

$$
\begin{aligned}
\delta_{B} g_{\mu \nu} & =-\delta \lambda\left[g_{\rho \nu} \partial_{\mu} c^{\rho}+g_{\rho \mu} \partial_{\nu} c^{\rho}+\partial_{\rho} g_{\mu \nu} c^{\rho}\right] \\
\delta_{B} c^{\mu} & =-\delta \lambda c^{\rho} \partial_{\rho} c^{\mu} \\
\delta_{B} \bar{c}_{\mu} & =i \delta \lambda B_{\mu} \\
\delta_{B} B_{\mu} & =0
\end{aligned}
$$

which is nilpotent. Here $\delta \lambda$ is an anticommuting parameter. We use the same gauge fixing as [1] using $\tilde{g}^{\mu \nu}$, whose BRST transformation is given by

$$
\delta_{B} \tilde{g}^{\mu \nu}=\delta \lambda\left(\tilde{g}^{\mu \rho} \partial_{\rho} c^{\nu}+\tilde{g}^{\nu \rho} \partial_{\rho} c^{\mu}-\tilde{g}^{\mu \nu} \partial_{\rho} c^{\rho}-\partial_{\rho} \tilde{g}^{\mu \nu} c^{\rho}\right) \equiv \delta \lambda \mathcal{D}_{\rho}^{\mu \nu} c^{\rho} .
$$

The gauge fixing term and Faddeev-Popov (FP) ghost terms are concisely written as

$$
\begin{aligned}
\mathcal{L}_{G F+F P} & =i \delta_{B}\left[\bar{c}_{\mu}\left(\partial_{\nu} h^{\mu \nu}-\frac{a}{2} B^{\mu}\right)\right] / \delta \lambda \\
& =-B_{\mu} \partial_{\nu} h^{\mu \nu}-i \bar{c}_{\mu} \partial_{\nu} \mathcal{D}^{\mu \nu}{ }_{\rho} c^{\rho}+\frac{a}{2} B_{\mu} B^{\mu}
\end{aligned}
$$

where $a$ is a gauge parameter and the indices are raised and lowered with the flat metric.

The simplest way to read off the propagator for the graviton is to first eliminate the auxiliary field $B^{\mu}$ and look at the quadratic part. We find that it is given by

$$
\begin{gathered}
\mathcal{L}_{2, t}=\frac{1}{4} h^{\mu \nu}\left[P^{(2)}(\beta \square+\sigma)+\frac{\square}{a} P^{(1)}+P^{(0, s)}\{(8 \alpha+3 \beta) \square-\sigma\}+2 P^{(0, w)}\left\{(8 \alpha+3 \beta) \square-\sigma+\frac{1}{a}\right\}\right. \\
\left.+\sqrt{2}\left(P^{(0, s w)}+P^{(0, w s)}\right)\{(8 \alpha+3 \beta) \square-\sigma\}\right]_{\mu \nu, \rho \sigma} \square h^{\rho \sigma},
\end{gathered}
$$

Using the completeness property and the orthogonality of the projection operators, we find the propagator is given by

$$
\begin{aligned}
D_{\mu \nu, \rho \sigma}(k)= & \frac{1}{(2 \pi)^{3}}\left[\frac{P^{(2)}}{k^{2}\left(\beta k^{2}-\sigma\right)}+\frac{P^{(0, s)}}{k^{2}\left\{(8 \alpha+3 \beta) k^{2}+\sigma\right\}}\right. \\
& \left.-\frac{a}{2 k^{2}}\left\{2 P^{(1)}+2 P^{(0, s)}+P^{(0, w)}-\sqrt{2}\left(P^{(0, s w)}+P^{(0, w s)}\right)\right\}\right]_{\mu \nu, \rho \sigma} .
\end{aligned}
$$

We shall take the Landau gauge given by $a=0$. Since the theory is invariant under the general coordinate transformation, this does not cause any problem, but simplifies the discussions considerably [1]. In this gauge, we have

$$
\partial_{\mu} h^{\mu \nu}=0 .
$$

Note that the above propagator satisfies $k^{\mu} D_{\mu \nu, \rho \sigma}(k)=0$ in this gauge. Also, the propagator damps as $k^{-4}$ for large momentum. As a result, as we argue later, the theory becomes renormalizable. However, there are two important exceptional cases: $8 \alpha+3 \beta=0$ and $\beta=0$. The former case corresponds to the new massive gravity recently discussed extensively [2]. Although this theory is found to be unitary either for $8 \alpha+3 \beta=0$ or $\beta=0$ [6]-[9], the following power counting argument fails precisely in these cases and the theory is not renormalizable. For $\sigma=0$, in addition to $8 \alpha+3 \beta=0$ discussed in [6], the propagator cannot be obtained in the usual sense, and the following argument for the renormalizability does not apply. 


\subsection{Slavnov-Taylor identity}

If we introduce the Grassmann-odd source $K_{\mu \nu}$ and the Grassmann-even source $L_{\mu}$, we have the BRST-invariant action

$$
\begin{aligned}
I_{\text {sym }}\left[h_{\mu \nu}, \bar{c}_{\alpha}, c^{\beta}, K_{\mu \nu}, L_{\rho}\right] & =\int d^{3} x\left[\mathcal{L}_{G M G}+\mathcal{L}_{G F+F P}+K_{\mu \nu} \mathcal{D}_{\rho \nu}^{\mu \nu} c^{\rho}-L_{\mu} c^{\nu} \partial_{\nu} c^{\mu}\right] \\
& \equiv \int d^{3} x \mathcal{L}_{\text {sym }} .
\end{aligned}
$$

The BRST invariance follows from (7), (9) and the nilpotency of the BRST transformation.

The generating functional of Green's functions is given by

$$
\begin{aligned}
Z\left[J_{\mu \nu}, \bar{\eta}_{\alpha}, \eta^{\beta}, K_{\mu \nu}, L_{\rho}\right] & =\int[d h][d \bar{c}][d c] \exp \left(i \int d^{3} x\left[\mathcal{L}_{s y m}+J_{\mu \nu} h^{\mu \nu}+\bar{\eta}_{\alpha} c^{\alpha}+\bar{c}_{\alpha} \eta^{\alpha}\right]\right) \\
& \equiv \exp \left(i W\left[J_{\mu \nu}, \bar{\eta}_{\alpha}, \eta^{\beta}, K_{\mu \nu}, L_{\rho}\right]\right)
\end{aligned}
$$

where $J_{\mu \nu}$, and $\bar{\eta}_{\alpha}$ and $\eta^{\alpha}$ are Grassmann-even and Grassmann-odd sources, respectively. The BRST invariance of the functional (14)

$$
0=\int[d h][d \bar{c}][d c] \delta_{B} \exp \left(i \int d^{3} x\left[\mathcal{L}_{s y m}+J_{\mu \nu} h^{\mu \nu}+\bar{\eta}_{\alpha} c^{\alpha}+\bar{c}_{\alpha} \eta^{\alpha}\right]\right)
$$

implies that

$$
\left\langle\int d^{3} x\left[J_{\mu \nu} \mathcal{D}^{\mu \nu}{ }_{\rho} c^{\rho}+\bar{\eta}_{\mu} c^{\nu} \partial_{\nu} c^{\mu}+i \frac{1}{a} \eta_{\mu} \partial_{\nu} h^{\mu \nu}\right]\right\rangle=0,
$$

where the field $B_{\mu}$ is eliminated by its field equation. This yields the Slavnov-Taylor identity

$$
\int d^{3} x\left[J_{\mu \nu} \frac{\delta W}{\delta K_{\mu \nu}}-\bar{\eta}_{\mu} \frac{\delta W}{\delta L_{\mu}}+\frac{i}{a} \eta^{\mu} \partial_{\nu} \frac{\delta W}{\delta J_{\mu \nu}}\right]=0 .
$$

The equations of motion for the FP ghost is

$$
\partial_{\nu} \frac{\delta W}{\delta K_{\mu \nu}}+i \eta_{\mu}=0
$$

As usual, the effective action is defined by

$$
\tilde{\Gamma}\left[h^{\mu \nu}, \bar{c}_{\alpha}, c^{\beta}, K_{\mu \nu}, L_{\rho}\right] \equiv W\left[J_{\mu \nu}, \bar{\eta}_{\alpha}, \eta^{\beta}, K_{\mu \nu}, L_{\rho}\right]-\int d^{3} x\left[J_{\mu \nu} h^{\mu \nu}+\bar{\eta}_{\alpha} c^{\alpha}+\bar{c}_{\alpha} \eta^{\alpha}\right] .
$$

It follows from (14) that

$$
h^{\mu \nu}=\frac{\delta W}{\delta J_{\mu \nu}}, \quad c^{\mu}=\frac{\delta W}{\delta \bar{\eta}_{\mu}}, \quad \bar{c}_{\mu}=-\frac{\delta W}{\delta \eta^{\mu}} .
$$

The relations dual to these are

$$
J_{\mu \nu}=-\frac{\delta \tilde{\Gamma}}{\delta h^{\mu \nu}}, \quad \bar{\eta}_{\alpha}=\frac{\delta \tilde{\Gamma}}{\delta c^{\alpha}}, \quad \eta^{\alpha}=-\frac{\delta \tilde{\Gamma}}{\delta \bar{c}_{\alpha}} .
$$

We further define

$$
\Gamma=\tilde{\Gamma}+\int d^{3} x \frac{1}{2 a}\left(\partial_{\nu} h^{\mu \nu}\right)^{2}
$$


With the help of the relations

$$
\frac{\delta \Gamma}{\delta K_{\mu \nu}}=\frac{\delta W}{\delta K_{\mu \nu}}, \quad \frac{\delta \Gamma}{\delta L_{\mu}}=\frac{\delta W}{\delta L_{\mu}}
$$

and the ghost field equation

$$
\partial^{\nu} \frac{\delta \Gamma}{\delta K_{\mu \nu}}-i \frac{\delta \Gamma}{\delta \bar{c}^{\mu}}=0
$$

the Slavnov-Taylor identity reduces to

$$
\int d^{3} x\left[\frac{\delta \Gamma}{\delta h^{\mu \nu}} \frac{\delta \Gamma}{\delta K_{\mu \nu}}+\frac{\delta \Gamma}{\delta c^{\mu}} \frac{\delta \Gamma}{\delta L_{\mu}}\right]=0
$$

The $n$-loop part of the effective action is denoted by $\Gamma^{(n)}$. The effective action is a sum of these terms:

$$
\Gamma=\sum_{n=0}^{\infty} \Gamma^{(n)}
$$

Suppose that we have successfully renormalized the effective action up to $(n-1)$-loop order. Write

$$
\Gamma^{(n)}=\Gamma_{\text {finite }}^{(n)}+\Gamma_{\text {div }}^{(n)}
$$

If we insert this breakup into Eq. (25) and keep only the terms which are of $n$-loop order, we get

$$
\begin{array}{r}
\int d^{3} x\left[\frac{\delta \Gamma_{\text {div }}^{(n)}}{\delta h^{\mu \nu}} \frac{\delta \Gamma^{(0)}}{\delta K_{\mu \nu}}+\frac{\delta \Gamma_{\text {div }}^{(n)}}{\delta c^{\mu}} \frac{\delta \Gamma^{(0)}}{\delta L_{\mu}}+\frac{\delta \Gamma^{(0)}}{\delta h^{\mu \nu}} \frac{\delta \Gamma_{\text {div }}^{(n)}}{\delta K_{\mu \nu}}+\frac{\delta \Gamma^{(0)}}{\delta c^{\mu}} \frac{\delta \Gamma_{\text {div }}^{(n)}}{\delta L_{\mu}}\right] \\
=-\int d^{3} x \sum_{i=0}^{n}\left[\frac{\delta \Gamma_{\text {finite }}^{(n-i)}}{\delta h^{\mu \nu}} \frac{\delta \Gamma_{\text {finite }}^{(i)}}{\delta K_{\mu \nu}}+\frac{\delta \Gamma_{\text {finite }}^{(n-i)}}{\delta c^{\rho}} \frac{\delta \Gamma_{\text {finite }}^{(i)}}{\delta L_{\rho}}\right] .
\end{array}
$$

Since each term on the right-hand side of (28) remains finite as $\epsilon(=d-3) \rightarrow 0$ in the dimensional regularization, while each term on the left-hand side contains a factor with a pole in $\epsilon$, each side of the equation must vanish separately. This leads to

$$
\int d^{3} x\left[\frac{\delta \Gamma^{(0)}}{\delta K_{\mu \nu}} \frac{\delta}{\delta h^{\mu \nu}}+\frac{\delta \Gamma^{(0)}}{\delta L_{\lambda}} \frac{\delta}{\delta c^{\lambda}}+\frac{\delta \Gamma^{(0)}}{\delta h^{\mu \nu}} \frac{\delta}{\delta K_{\mu \nu}}+\frac{\delta \Gamma^{(0)}}{\delta c^{\lambda}} \frac{\delta}{\delta L_{\lambda}}\right] \Gamma_{\operatorname{div}}^{(n)}=0 .
$$

This identity will be used in later discussions of renormalizability.

\subsection{Renormalizability}

Under the expansion (2), the Einstein term gives graviton vertices with two derivatives, and curvature square terms give those with four derivatives. Consider arbitrary Feynman diagrams. We use the following notations.

$V_{2}$ : the number of graviton vertices with two derivatives from the $R$ term.

$V_{4}$ : the number of graviton vertices with four derivatives from the $R^{2}$ term. 
$V_{c}$ : the number of ghost-antighost-graviton vertices with two derivatives.

$V_{K}$ : the number of $K$-graviton-ghost vertices.

$V_{L}$ : the number of $L$-ghost-ghost vertices.

$I_{h}$ : the number of internal-graviton propagators.

$I_{c}$ : the number of internal-ghost propagators.

$E_{h}$ : the number of external gravitons.

$E_{c}$ : the number of external ghosts.

Since the graviton propagator behaves as $k^{-4}$ and the FP ghost propagator as $k^{-2}$, we are led by the standard power counting to the degree of divergence of an arbitrary diagram:

$$
D=3 L-4 I_{h}-2 I_{c}+4 V_{4}+2 V_{2}+2 V_{c}+V_{K}+V_{L} .
$$

Using the relation

$$
L=I_{h}+I_{c}-\left(V_{4}+V_{2}+V_{c}+V_{K}+V_{L}-1\right),
$$

we get

$$
D=3-I_{h}+I_{c}+V_{4}-V_{2}-V_{c}-2 V_{K}-2 V_{L} .
$$

We further use the topological relation

$$
2 V_{c}+V_{K}+2 V_{L}=2 I_{c}+E_{c}+E_{\bar{c}}
$$

to obtain

$$
D=3-\left(I_{h}-V_{4}\right)-V_{2}-\frac{3}{2} V_{K}-V_{L}-\frac{1}{2}\left(E_{c}+E_{\bar{c}}\right) .
$$

Now the ghost vertex contained in the FP ghost term in (9), upon partial integration, can be rewritten as

$$
i\left[\partial_{\rho} \partial_{\mu} \bar{c}_{\nu} \cdot c^{\nu} h^{\mu \rho}+\partial_{\mu} \bar{c}_{\nu} \cdot c^{\nu} \partial_{\rho} h^{\mu \rho}+\partial_{\mu} \bar{c}_{\nu} \cdot c^{\mu} \partial_{\rho} h^{\nu \rho}\right] .
$$

In the Landau gauge in which we have (12), the last two terms do not couple to the propagator. Also integration by parts in the remaining term can be used to move the derivative onto the ghost using the gauge condition:

$$
i \partial_{\rho} \partial_{\mu} \bar{c}_{\nu} \cdot c^{\nu} h^{\mu \rho} \approx i \bar{c}_{\nu} \partial_{\rho} \partial_{\mu} c^{\nu} h^{\mu \rho} .
$$

As a result, in one-particle irreducible (1PI) diagrams, each external ghost and antighost carries two factors of external momentum [1]. The resulting degree of divergence of an arbitrary 1PI diagram is then

$$
D^{(1 P I)}=3-\left(I_{h}-V_{4}\right)-V_{2}-\frac{3}{2} V_{K}-V_{L}-\frac{5}{2}\left(E_{c}+E_{\bar{c}}\right) .
$$

We note that $I_{h}-V_{4} \geq 0$ for 1PI diagrams. Consequently we find that the possible divergences are restricted; those with external ghosts and antighosts have $D^{(1 \mathrm{PI})} \leq-2$, those with the external $K$ and ghost $D^{(1 \mathrm{PI})} \leq-1$, and those with $L$ and two ghosts have $D^{(1 \mathrm{PI})} \leq-3$. Hence, we have

$$
\frac{\delta \Gamma_{\text {div }}^{(n)}}{\delta c^{\lambda}}=\frac{\delta \Gamma_{\text {div }}^{(n)}}{\delta K^{\mu \nu}}=\frac{\delta \Gamma_{\text {div }}^{(n)}}{\delta L_{\lambda}}=0 .
$$


The Slavnov-Taylor identity (29) then reduces to

$$
\int d^{3} x \frac{\delta \Gamma^{(0)}}{\delta K^{\mu \nu}} \frac{\delta \Gamma_{\mathrm{div}}^{(n)}}{\delta \tilde{g}^{\mu \nu}}=0 .
$$

Together with (38), this implies that $\Gamma_{\text {div }}^{(n)}$ is gauge invariant. Consequently $\Gamma_{\text {div }}^{(n)}$ are local gauge-invariant functionals of $\tilde{g}^{\mu \nu}$ with zero and two derivatives (up to three). This allows only the counterterms of the Einstein and the cosmological terms. Hence the theory is superrenormalizable.

However we note that there are very important exceptional cases to these arguments: $8 \alpha+$ $3 \beta=0$ and $\beta=0$. As noted in Sec. 2.2, the behavior of the propagator becomes $k^{-2}$ even in the Landau gauge and the degree of divergence is changed to

$$
D^{(1 P I)}=3+I_{h}+V_{4}-V_{2}-\frac{3}{2} V_{K}-V_{L}-\frac{5}{2}\left(E_{c}+E_{\bar{c}}\right) .
$$

Diagrams are more divergent as the numbers of the vertices and propagators of the graviton are increased, and the theory is not renormalizable.

\section{Discussions and conclusions}

In this paper we have studied whether higher derivative gravity in three dimensions is renormalizable or not. As it turns out, the general theory is renormalizable, but if we restrict the coefficients to special values $8 \alpha+3 \beta=0$ or $\beta=0$, then the convergence property of the graviton propagator becomes worse, and the power counting indicates that the theory is not renormalizable. Unfortunately, these are precisely the cases when the theories are unitary at the tree level; the former case is known as new massive gravity, and the latter is a special case of $f(R)$ gravity.

In new massive gravity, it is known that the condition $8 \alpha+3 \beta=0$ eliminates the propagating spin 0 mode. From the graviton propagator (11), we see that it is the spin 0 component of the graviton that gives this bad behavior to the propagator. If the scalar mode decouples from all physical quantities, then this would not spoil the renormalizability of the theory. However, this is not the case. Even if the scalar mode is excluded in the initial states, this mode enters physical processes through the interactions so that it produces divergences which cannot be renormalized. Only if there is some kind of local gauge invariance to ensure the decoupling of the scalar mode can the theory be renormalizable. However, there is no such invariance in our present higher derivative gravity in three dimensions and the theory is not renormalizable with the above restriction on the coefficients.

There has been an argument that claims the new massive gravity is renormalizable [12]. We find that there are two points that need careful consideration. The first is that the author introduces mass terms which break the gauge invariance of the theory and derives the graviton propagator, which has bad behavior and makes the resulting power counting invalid. However, it was argued that these parts decouple and one can ignore these bad behaviors; only the $k^{-4}$ behavior of the spin 2 component is taken. This does not seem to be justified because there is no invariance that ensures the decoupling of the scalar mode in this theory when interactions are taken into account. Moreover the gauge invariance or the invariance under the general coordinate transformation is explicitly broken by the mass term, and it is expected that there arises an inconsistency in the spin-2 part with nonlinear interactions. Second, the author claims that the massless limit gives the new massive gravity so that the latter itself is renormalizable. 
However the massless limit is subtle in general field theories, and we expect that this procedure is dubious. If such manipulation were allowed, we could have proved the renormalizability of the Yang-Mills theory including the mass term. Considering these points, we believe that this "proof" cannot be justified and conclude that the new massive gravity is not renormalizable though the general theory is. Thus unfortunately the new massive gravity does not give the quantum thoery of gravity, but our analysis opens the possibility that if there is some symmetry which can eliminate the scalar mode, there is a possibility of finding renormalizable and unitary theory.

\section{Acknowledgement}

We would like to thank S. Deser, A. Ishibashi and P. K. Townsend for very useful discussions. This work was supported in part by the Grant-in-Aid for Scientific Research Fund of the JSPS (C) No. 20540283, No. 21. 09225 and (A) No. 22244030.

\section{References}

[1] K. S. Stelle, Phys. Rev. D 16 (1977) 953.

[2] E. A. Bergshoeff, O. Hohm and P. K. Townsend, Phys. Rev. Lett. 102 (2009) 201301 [arXiv:0901.1766 [hep-th]].

[3] S. Deser, R. Jackiw and S. Templeton, Annals Phys. 140 (1982) 372 [Erratum-ibid. 185 (1988) 406]

[4] Y. Liu and Y. W. Sun, Phys. Rev. D 79 (2009) 126001 [arXiv:0904.0403 [hep-th]].

[5] M. Nakasone and I. Oda, Prog. Theor. Phys. 121 (2009) 1389 [arXiv:0902.3531 [hep-th]].

[6] S. Deser, Phys. Rev. Lett. 103 (2009) 101302 [arXiv:0904.4473 [hep-th]].

[7] I. Gullu, T. C. Sisman and B. Tekin, Phys. Rev. D 81 (2010) 104017 [arXiv:1002.3778 [hep-th]].

[8] E. A. Bergshoeff, O. Hohm and P. K. Townsend, Phys. Rev. D 79 (2009) 124042 [arXiv:0905.1259 [hep-th]].

[9] N. Ohta, Class. Quant. Grav. 29 (2012) 015002 [arXiv:1109.4458 [hep-th]].

[10] H. Nishino and S. Rajpoot, Phys. Lett. B 639 (2006) 110 [arXiv:hep-th/0607241].

[11] S. Deser and Z. Yang, Class. Quant. Grav. 7 (1990) 1603.

[12] I. Oda, JHEP 0905 (2009) 064 [arXiv:0904.2833 [hep-th]].

[13] E. A. Bergshoeff, O. Hohm, P. K. Townsend, arXiv:1007.4561 [hep-th]. 